\title{
Erratum to: Acute lymphoblastic leukemia and Down syndrome: the collaborative study of the Tokyo Children's Cancer Study Group and the Kyushu Yamaguchi Children's Cancer Study Group
}

\author{
Hiroaki Goto $\cdot$ Takeshi Inukai $\cdot$ Hiroyasu Inoue $\cdot$ Chitose Ogawa $\cdot$ Takashi Fukushima Miharu Yabe \\ Akira Kikuchi · Kazutoshi Koike • Keitaro Fukushima • Keiichi Isoyama • Tomohiro Saito • Akira Ohara • \\ Ryoji Hanada $\cdot$ Jiro Iwamoto $\cdot$ Noriko Hotta $\cdot$ Yoshihisa Nagatoshi · Jun Okamura $\cdot$ Masahiro Tsuchida
}

Published online: 9 March 2011

(c) The Japanese Society of Hematology 2011

\section{Erratum to: Int J Hematol (2011) 93:192-198 \\ DOI 10.1007/s12185-011-0765-3}

This article originally was published without including the following Acknowledgment:

This study was supported by a grant from Children's Cancer Association of Japan.

The online version of the original article can be found under doi: $10.1007 / \mathrm{s} 12185-011-0765-3$.

\section{H. Goto ( $\square)$}

Department of Pediatrics, Yokohama City University,

School of Medicine, 3-9 Fukuura Kanazawa-ku,

Yokohama 236-0004, Japan

e-mail: hgoto39@med.yokohama-cu.ac.jp

T. Inukai

Department of Pediatrics, University of Yamanashi,

School of Medicine, Kohu, Japan

H. Inoue

Division of Oncology,

Kanagawa Children's Medical Center, Yokohama, Japan

C. Ogawa

Department of Pediatrics,

St. Luke's International Hospital, Tokyo, Japan

T. Fukushima

Department of Pediatrics, School of Medicine,

University of Tsukuba, Tsukuba, Japan

M. Yabe

Department of Cell Transplantation,

Tokai University Hospital, Isehara, Japan

A. Kikuchi

Department of Pediatrics, Faculty of Medicine,

University of Tokyo, Tokyo, Japan
K. Koike $\cdot$ M. Tsuchida

Department of Pediatric Hematology and Oncology,

Ibaraki Children's Hospital, Mito, Japan

\section{K. Fukushima}

Department of Pediatrics, Dokkyo Medical University,

Mibu, Japan

K. Isoyama

Department of Pediatrics, Showa University,

School of Medicine, Fujigaoka Hospital, Yokohama, Japan

T. Saito

Department of Health Policy, National Center for Child Health and Development, Tokyo, Japan

\section{A. Ohara}

Department of First Pediatrics, Toho University Medical Center,

Oomori Hospital, Tokyo, Japan

R. Hanada

Department of Hematology/Oncology,

Saitama Children's Medical Center, Iwatsuki, Japan

J. Iwamoto

Department of Pediatrics, Aso-Iizuka Hospital, Iizuka, Japan

N. Hotta

Department of Pediatrics, Yamaguchi University Graduate

School of Medicine, Yamaguchi, Japan

Y. Nagatoshi · J. Okamura

Section of Pediatrics, National Kyushu Cancer Centre,

Fukuoka, Japan 